\title{
Transatlantica
}

Revue d'études américaines. American Studies Journal

\section{Natures mourantes / Rethinking Still Lifes}

Université Toulouse 2-Jean Jaurès, 20 mars 2021

\section{Ana Calvete et Frank Miroux}

\section{(2) OpenEdition}

Journals

Édition électronique

URL : https://journals.openedition.org/transatlantica/16599

DOI : 10.4000/transatlantica. 16599

ISSN : $1765-2766$

Éditeur

Association française d'Etudes Américaines (AFEA)

Référence électronique

Ana Calvete et Frank Miroux, « Natures mourantes / Rethinking Still Lifes », Transatlantica [En ligne], 1 । 2021, mis en ligne le 22 juin 2021, consulté le 02 février 2023. URL : http://journals.openedition.org/ transatlantica/16599; DOI : https://doi.org/10.4000/transatlantica.16599

Ce document a été généré automatiquement le 2 février 2023

\section{(c) (†) $\odot$}

Creative Commons - Attribution - Pas d'Utilisation Commerciale - Pas de Modification 4.0 International - CC BY-NC-ND 4.0

https://creativecommons.org/licenses/by-nc-nd/4.0/ 


\title{
Natures mourantes / Rethinking Still Lifes
}

Université Toulouse 2-Jean Jaurès, 20 mars 2021

\author{
Ana Calvete et Frank Miroux
}

1 La journée d'études "Natures mourantes ", organisée par Claire Cazajous-Augé sous l'égide du laboratoire Cultures anglo-saxonnes (Université Toulouse 2-Jean Jaurès), s'est tenue le 20 mars 2021 sous forme de webinaire. Claire Cazajous-Augé a ouvert la journée en invitant à une réflexion sur la vitalité et la vulnérabilité des corps, qui nous renvoient à la mort comme à un événement partagé entre les espèces et fonctionnent comme métonymie de leur disparition.

\section{David Vann (écrivain), "Why not bury the man with the buck's head? »}

David Vann, écrivain né en Alaska, propose dans sa conférence plénière une lecture commentée d'extraits de son roman Goat Mountain, publié en 2013.

La première scène s'inspire d'un épisode de sa jeunesse au Canada et narre les étapes de la mise à mort d'un cervidé par Tom, un garçon de onze ans. La bête, paralysée par une balle, n'en finit pas de mourir. Le jeune garçon obéit à un rituel familial en achevant l'animal blessé au couteau. Le narrateur remonte au meurtre d'Abel par Caïn et affirme qu'il faut regarder le meurtre en face car il fait partie des instincts premiers. Le cervidé devient un sacrifice présenté à la famille et à Dieu. Après sa mort, le récit suit les mains de Tom qui découvrent et coupent les organes internes de l'animal. Dans une libation rituelle et obligatoire, il mange une partie du foie, qui a pour lui « le goût des entrailles de tout homme et de toute bête ». Alors qu'il pense " [j]'étais redevenu bête », son père et son grand-père le déclarent "maintenant, un homme ». Vann explique que l'enfant n'est pas encore figé dans son identité, qu'il peut encore imaginer devenir animal. S'ensuit la lecture d'une nuit passée à traîner la carcasse jusqu'au camp, où Tom la pend à un arbre à côté de la dépouille d'un braconnier qu'il a précédemment tué. Le grand- 
père et le père se disputent à propos de la valeur morale de ce geste car, pour le premier, le braconnier est semblable à l'animal.

4 Vann discute ensuite de ses choix narratifs avec Claire Cazajous-Augé. Il relie la Sainte Trinité familiale du récit à ses origines Cherokee et aux dommages que les missionnaires ont infligés à cette tribu. En ce qui concerne l' « horreur» de la mise à mort, il ne s'agit pas, comme dans les films d'épouvante, de découper le corps en morceaux et de détacher la violence de toute psychologie. L'auteur explique qu'il vise plutôt un effet dramatique créé par la représentation d'une violence chargée émotionnellement et psychologiquement. Dans la description esthétisée du corps mourant du cervidé, c'est aussi la beauté qu'il recherche.

Quand Cazajous-Augé l'interroge sur sa syntaxe elliptique et son recours aux phrases nominales, l'auteur renvoie à la double origine de la langue anglaise, à la fois brutale et saxonne, et raffinée et française. Selon lui, l'héritage germanique est plus apte à décrire ce qui reste lorsqu'on prive l'être humain de son raffinement et qu'on essaie d'une part de remonter au moment où il s'est vu différent de l'animal et, d'autre part, d'imaginer l'esprit plus primal qui accomplit les gestes de prédation depuis des millénaires.

6 Enfin, Vann explique que l'environnement joue un rôle primordial dans sa méthode: quand il élabore une fiction, il commence par la description d'un lieu naturel qui révèle la psychologie du protagoniste qui s'y insèrera. L'histoire prend alors forme.

\section{Mathilde La Cassagnère (Université Savoie Mont Blanc), « Poems from the interval: animal violence and animal still-lifes in the art of Ted Hughes »}

7 L'atelier de la matinée, « l'art de la décomposition », s'ouvre sur l'analyse par Mathilde La Cassagnère de l'animal mourant dans les textes du poète anglais Ted Hughes. Elle étudie la vitalité des animaux mourants à la lumière des rituels chamaniques qui fascinaient le poète. Le chaman, dans un état de trance, invoque un animal-démon qui lui fait vivre une mort magique et le démembre pour qu'il puisse renaître dans un corps d'animal. La Cassagnère met en évidence la lutte des regards entre l'homme et l'animal. Loin d'être pétrifiée par le regard de l'homme, la bête prend vie et le poème permet une forme de gestation où se mêlent les règnes humains et animaux dans ce que Donna Haraway appellerait une « figure ». La mort, événement partagé par les espèces, occupe cet espace liminal et constitue une troisième voie pour comprendre le monde, à l'instar $\mathrm{du}$ bardo, état méditatif et intermédiaire du bouddhisme tibétain, qui permet au chaman de faire l'expérience d'une mort extatique.

8 À travers l'interprétation des poèmes " The Jaguar ", "Pike » et "Second Glance at the Jaguar ", La Cassagnère présente une lecture de l'œuvre de Hughes selon trois axes, étudiant d'abord la scopophilie inversée à l'œuvre dans ses textes, puis l'entrelacement de l'homme et de l'animal et enfin la mort en tant qu'expérience régénératrice. Dans "Pike ", par exemple, l'anthropomorphisation des bêtes appelle à un questionnement sur les comportements animaux et prédateurs des êtres humains. Toutefois, tantôt dévorés par l'œil humain, tantôt plongeant dans cet œil qui les observe, les brochets nous rendent nos regards de lecteurs. Le poème donne naissance à une perspective « pikanthropique » car il naît du texte une créature entre brochet et humain, entre la mort donnée et la mort reçue. Le poème cultive l'ambiguïté, permettant des relectures 
multiples où ce n'est plus le brochet qui est "pêché", mais l'homme qui devient poisson. La danse des brochets évoque la trance du chaman. L'absorption est mutuelle et l'homme cesse finalement d'être dévorant pour se laisser « habiter par l'intelligence charnelle de la créature ", comme l'a formulé David Abram dans Becoming Animal.

Pour conclure, La Cassagnère suggère que les poèmes de Ted Hughes délivrent de la «profane» condition humaine en permettant au lecteur comme au chaman de retourner à leurs os, c'est-à-dire à une source de vie.

\section{Ariane Carmignac (Université de Lille), « Des natures inquiètes »}

10 Ariane Carmignac aborde l'art contemporain comme transformation du mourant, devenu matière première qui reprend vie, comme en témoigne le poisson ficelé de Véronique Ellena. Elle interroge ce que nous percevons comme insoutenable. Partant de représentations qu'elle définit comme "limites", elle examine l'appropriation artistique des «bas morceaux » et s'intéresse d'abord à la rhyparographie, c'est-à-dire la représentation des restes, des objets insignifiants et dégoûtants. Elle se penche ensuite sur la peau des images et le regard dessillé et parodique qu'elles posent sur l'organique, avant d'aborder la vitalité des natures mourantes.

11 Sous-genre de la nature morte, la rhyparographie trouve sa source dans l'Antiquité, comme le montre la description que fait Pline de La Chambre non balayée de Sôsos de Pergame, qui donne à voir des déchets tombés à terre lors d'un banquet. Carmignac attire l'attention de l'auditoire sur d'autres exemples tels que les animaux figés dans les assiettes de Bernard Palissy, céramiste du $\mathrm{XvI}^{\mathrm{e}}$ siècle, dont la rumeur disait qu'il les avait moulés vivants dans un acte prédateur. À l'instar d'André Bazin, qui parle d'un "complexe de la momie » pour expliquer les velléités de préservation de l'art, cette communication nous invite à regarder le référent comme quelque chose qui adhère à sa représentation photographique.

12 Les images de l'organique mourant agissent comme memento mori, tout comme le processus entropique à l'œuvre dans l'atelier de l'artiste. La rhyparographie possède aussi une dimension parodique et lyrique illustrée entre autres par Joachim Mogarra, qui saisit le lyrisme de la peau en spirale d'un fruit épluché («Spiral Jetty»). Les artistes nous donnent une "éprouvante leçon d'anatomie », explique Carmignac, qui cite notamment l'œuvre d'Yves Trémorin, dont le lapin dépecé reproduit L'Origine $d u$ monde, rendant repoussant le motif éculé. Enfin, elle évoque la nouvelle d'Italo Calvino "L'aventure d'un photographe », qui met en scène un artiste qui trouve l'absolu en détruisant ses clichés photographiques puis en en photographiant les morceaux épars, suggérant ainsi la capacité de la nature mourante à renaître des rebuts.

\section{Vincent Lecomte (Université de Saint-Étienne), « La bête mise à mort : brève généalogie d'une projection »}

13 Dans cette communication Vincent Lecomte analyse la représentation en 2007 de la mise à mort d'un chien par Guillermo Vargas Jimenez, dit « Habacuc », dans une galerie du Nicaragua. Cette œuvre pointe du doigt le sort réservé à l'animal, dont l'exécution est évacuée de la scène sociale et possède une visée politique puisqu'elle est 
accompagnée de la combustion de crack et de marijuana et de l'hymne sandiniste diffusé à l'envers. Le chien errant, décharné, est attaché dans une pièce où déambule le public, qui ne tente à aucun moment de le nourrir. Vargas affirme qu'il était de toute façon condamné et que l'œuvre sensibilise au sort des chiens des rues.

Il intègre dans la performance l'indignation du public, largement diffusée sur internet, et dénonce la propagation du jugement dans une société hypermédiatisée, ainsi que la complicité passive des internautes avec les images obscènes qu'ils consomment. Il faut cependant noter que l'œuvre a été qualifiée de «hoax» car il n'existe pas de preuves tangibles de la mort du chien.

Cette démarche révèle notre indifférence aux autres espèces et aux exclus et rend leur valeur à ces autres, par le biais du nom "Natividad", donné à l'œuvre et au chien au seuil de la mort, dans un geste de rachat grotesque et dérisoire. Une toxicomane du même nom avait été tuée au Nicaragua par des rottweilers, ce qui suggère que la mort du chien fait écho à celle des habitants miséreux. Le dispositif est à la fois morbide et régénérant: sur le mur de la salle où se trouve le chien, l'inscription en croquettes « lo que eres lees", "vous êtes ce que vous lisez ", se trouve juste hors de sa portée. Par cette inscription, Vargas entend réveiller d'une lecture passive. Il rend corps au verbe, qui a été désincarné lorsque le vivant a été réduit à de la matière. L'animal, dans l'œuvre de Vargas, oblige le public à se racheter au chevet d'une nature mourante.

L'après-midi a consisté en deux conférences plénières entre lesquelles s'est intercalé un atelier organisé autour de problématiques d'ordre environnemental.

\section{Anne Simon (EHESS, CNRS), « Royaume de la mort : de l'abattoir en littérature »}

Anne Simon s'intéresse à ce qu'elle nomme la littérature agroalimentaire, c'est-à-dire une littérature qui aborde les modes industriels de production de la viande (élevages intensifs et abattoirs). Son intervention s'inscrit donc à contre-courant de certains constats dressés lors des sessions matinales à propos de l'entrelacement entre le mort et le vivant. En effet, dans les textes sur lesquels elle se penche, les univers évoqués n'ont plus rien de naturel et excluent d'emblée toute promesse de vie.

Dans une première partie, Simon met en avant le caractère essentiel de cette littérature qui donne à voir ce qui a fait l'objet d'une élision, à savoir la souffrance animale et la mise à mort. Elle démontre par l'étude d'extraits choisis que certains auteurs mettent en œuvre des stratégies narratives visant à restituer leur visibilité à ces lieux qu'on tente pourtant de soustraire aux yeux du public. Elle insiste également sur le fait que pour certains de ces auteurs, l'esthétisation de la mort et de la souffrance animales constitue un moyen efficace de rendre compte de l'horreur des scènes décrites.

La conférence se poursuit sur le constat d'une conjonction intéressante entre la mise au secret de ces lieux de mort et le gigantisme croissant qui caractérise cette industrie. Simon propose donc une réflexion sur l'abstraction mathématique et l'hyper matérialité organique dans les récits agroalimentaires. Il en ressort que le phénomène de spatialisation monstrueuse qui opère dans ces textes situe souvent la représentation 
hors des normes humaines. Les chiffres prennent alors le relai des mots pour évoquer l'irreprésentable et l'impensable. Simon identifie également dans ces récits le retour quasi systématique d'une naturalité démoniaque, d'une nature acide, tandis que les odeurs de fluides corporels et de déjections envahissent l'espace du texte. Les déchets produits par la mort du vif débordent alors du périmètre auquel on avait tenté de circonscrire la mort. Le processus de la mort mécanisée échappe alors à tout contrôle, ce qui signe souvent la destruction de ces lieux d'abattage.

Pour conclure, Simon aborde le rôle de la littérature agroalimentaire dans la construction d'un langage de la mort du vivant. Elle évoque les nomenclatures industrielles qui portent atteinte non seulement au vivant, mais aussi au langage, et ce à trois niveaux. Tout d'abord, on constate une inversion du langage, qui cesse alors de constituer une langue vive pour devenir une novlangue. Dans un deuxième temps, il s'agit d'une atteinte aux humains qui, par le biais du langage, sont transformés en automates. Enfin, subsiste la question de l'animal dénommé - mal nommé -, une atteinte à la justesse de la langue dès lors que l'animal perd sa spécificité étymologique et donc radicale : l'animation. C'est précisément ce statut d'être animé que les écrivains abordés tentent de restituer aux animaux à travers leurs récits. C'est donc d'une littérature qui participe de la réparation du monde en réparant la langue abîmée qu'il s'agit. D'une littérature qui, comme Simon le précise en guise de conclusion, oppose à la thanatopoétique industrielle une zoopoéthique redonnant à l'humain son humanité et à l'animal son animalité.

\section{Marie Cazabon-Mazerolles (Université de Poitiers), «'From Zoo to Bot' : putréfaction de l'animal humain et transcendance écologique dans Being Dead de Jim Crace »}

21 Marie Cazabon-Mazerolles se penche sur la notion de transcendance écologique dans le roman Being Dead, de l'écrivain britannique Jim Crace. S'agissant d'un ouvrage où le lecteur assiste à la lente putréfaction des corps d'un couple de zoologistes assassinés dès le début du roman, Cazabon-Mazerolles tente de cerner la manière dont Crace parvient à construire la figure du cadavre animé, de la dépouille durative. Elle insiste sur l'existence dans le texte de longues sections consacrées à la description du devenir des cadavres, procédé par le biais duquel l'auteur établit la persistance d'une animation strictement organique venant prendre le relais de l'existence consciente des personnages.

Selon elle, ce choix témoigne de la volonté de Crace de soutenir une vision matérialiste stipulant non que quelque chose survit au corps, mais que c'est par le corps qu'une forme de survivance advient. Ce faisant, il transcende la peur souvent incontrôlable qu'éprouve l'être humain à l'idée de faire l'objet d'une prédation, sa peur d'être à son tour consommé.

En outre, la condition post mortem des personnages relève autant de la décomposition que de la recomposition. Ainsi, les nombreux animaux qui viennent se nourrir sur les dépouilles enclenchent un cycle qui assure un devenir intramondain aux personnages. Leurs corps se trouvent non pas anéantis par ce processus de désintégration, mais disséminés dans les différents réseaux trophiques du monde naturel. 
Pour conclure, Cazabon-Mazerolles évoque une œuvre qui renouvelle radicalement la tradition du récit consolatoire en suggérant que c'est grâce à sa part la plus « animale » que l'humain peut accéder à une forme de transcendance - bioécologique, immanente et athée.

\section{Antoine Traisnel (Université du Michigan), « Thoreau's garden politics »}

Antoine Traisnel propose une communication portant sur un aspect émergeant de la recherche dans le domaine des humanités, la question des plantes, plus précisément du lien entre notre indifférence vis-à-vis de la condition végétale et les désastres climatiques et écologiques que nous subissons. Appuyant sa réflexion sur des références théoriques empruntées aux domaines de l'anthropologie, de l'écologie, de la philosophie et de la biopolitique, il constate d'emblée une mise en opposition fréquente des questions liées au bien-être animal et de celles relevant du monde végétal. La problématique qu'il dégage concerne la capacité de la biopolitique à intégrer le monde végétal à ses sujets d'étude. Selon lui, cela pourrait être un moyen de proposer des éléments de réflexion sur des problèmes plus vastes, tels que le devenir des populations humaines dont les modes de vie et les cultures ont souffert du manque de considération accordé au monde végétal.

Par le biais de références empruntées à l'ouvrage Walden, de Henry David Thoreau, Traisnel s'inscrit en faux contre l'idée selon laquelle la pensée philosophie occidentale $\mathrm{du}$ XIX ${ }^{\mathrm{e}}$ siècle a totalement ignoré la question des plantes. Derrière ce qu'on pourrait appeler la phytopoéthique de Thoreau, se dessine une politique de la plantation qui, bien que spécifique à une époque et à un espace géographique, pourrait servir à définir de nouveaux modèles permettant au monde végétal de trouver sa place au sein de la pensée biopolitique.

\section{Rick Bass (écrivain), « Beyond symbolism, beyond metaphor. wild animals as gods in a burning world "}

La conférence de l'écrivain américain Rick Bass vient conclure cette journée d'études. Bass s'appuie sur ses connaissances dans le domaine de la géologie pour souligner le paradoxe de l'être humain, qui se pense indestructible, mais dont l'histoire géologique du monde montre que sa condition d'omnivore géant l'expose à un risque d'extinction accru. Il s'attache ensuite à rétablir l'importance de ce qu'il nomme les demi-dieux. Il ne s'agit pas du dieu unique que l'être humain a souvent conçu à son image, mais des animaux, totems séculaires dont il précise qu'ils sont les jardiniers de la planète. Par ailleurs, c'est précisément dans cette interdépendance entre la faune et les écosystèmes, dans la complexité de la relation qui les lie, que Bass situe la beauté du monde.

Le romancier souligne l'erreur de Charles Darwin, qui, lors de son séjour aux Galapagos, n'a pas perçu la jeunesse d'un réseau trophique qu'il a pris pour exemple en dépit de son caractère immature. Selon Bass, les conclusions du naturaliste ont contribué à consolider un sens de la supériorité de l'espèce humaine, idée fondée sur la notion de descendance avec modification mise en avant par Darwin dans L'origine des espèces. 
Bass insiste alors sur l'équilibre qui caractérise les systèmes parvenus à maturité au sein desquels la biodiversité empêche toute espèce de prendre le dessus dès lors que la pérennité de ces systèmes repose sur un processus de coopération. La vallée du Yaak, dans le Montana, où Bass réside, constitue un parfait exemple de la complexité, mais aussi de la fragilité de ces réseaux.

Bass explique ensuite que son intérêt pour les animaux en tant qu'écrivain répond à un besoin d'observer une nature dont l'être humain doit tirer des enseignements s'il veut parvenir à survivre. Pour lui, les demi-dieux offrent en effet un potentiel créatif inépuisable, bien supérieur à celui qu'offre l'observation des humains.

Le verbe à la fois poétique, élégant et incisif de Rick Bass a clos une journée d'études qui a ouvert des pistes de réflexion prometteuses sur les représentations des «natures mourantes", mais aussi sur l'investissement éthique, voire politique, associé à ces questions. Des sujets sur lesquels il est d'autant plus urgent de réfléchir que, comme l'ont montré les différents intervenants, nature mourante et humanité déclinante vont nécessairement de pair.

\section{AUTEURS}

\section{ANA CALVETE}

Toulouse 2-Jean Jaurès \& Université d'Helsinki

FRANK MIROUX

Toulouse 2-Jean Jaurès 